\title{
HIGH FREQUENCY LOW IMPEDANCE CAPACITIVE SILICON BAR STRUCTURES
}

\author{
Siavash Pourkamali and Farrokh Ayazi \\ School of Electrical and Computer Engineering \\ Georgia Institute of Technology, Atlanta, GA 30332 \\ E-mail: siavash@ece.gatech.edu, Tel: (404)385-6693, Fax: (404)385-6650
}

\begin{abstract}
This paper presents high frequency high-Q micromachined capacitive silicon bulk acoustic resonators (SiBAR) with impedances well within the required range for electronic applications. Resonator motional resistances as low as $200 \Omega$ and quality factors as high as 90,000 are demonstrated for clampedclamped silicon BARs in the VHF range. The same resonators have been operated in their higher width extensional modes up to the fifth mode demonstrating frequencies as high as $765 \mathrm{MHz}$ with comparatively low motional resistances. Temperature characteristics and thermal temperature compensation techniques for such resonators are investigated. Thermal frequency tuning of $>1 \%$ is demonstrated for the high frequency resonators. Several structural variations of ultra-long silicon BARs for maximized electromechanical coupling are demonstrated and discussed as well.
\end{abstract}

\section{INTRODUCTION}

Development of silicon micromachined devices for frequency referencing is a fast growing technology with strong potential for a wide range of applications in electronics. High frequency capacitive silicon resonators have shown great capability for implementation of highly stable integrated frequency references $[1,2]$. Temperature compensated bulk micromachined single crystal silicon I-BARs operating in a few $\mathrm{MHz}$ range with quality factors in excess of 100,000 have been previously demonstrated [1].

For frequencies in the $100 \mathrm{MHz}$ and higher, capacitive electromechanical transduction may quickly become inefficient to provide satisfactory motional resistances. In addition, as the frequency increases, parasitic capacitances become a more serious issue and lower resistances are desired to alleviate excessive signal loss.

Thick capacitive bulk acoustic wave silicon resonators known as "SiBAR" were introduced in [3] for the first time. With their large transduction area and HARPSS-enabled deep-submicron capacitive gaps $[4,5]$, such resonators demonstrated much lower equivalent motional resistances $(\sim 5.0 \mathrm{k} \Omega)$ in the VHF range compared to disk resonators [4,5]. In this work, more efficient designs of SiBAR structures have enabled reduction of resonator impedances by another order of magnitude compared to values presented in [5]. Resulting motional resistances easily satisfy the requirements for low power VHF oscillators without the need for brute force pushing of the fabrication limits.

A large tuning range can be achieved by heating the high frequency SiBARs. Accordingly, a self compensating strategy can be developed that uses the resonator as a heater and a temperature sensor simultaneously.

Finally, several structural variations of SiBARs can be deployed to maximize their effective length and further increase the electromechanical coupling. Long SiBARs need to be supported at several locations along their structure to maintain reasonable structural stiffness.

\section{LOW IMPEDANCE CLAMPED-CLAMPED SiBARs}

HARPSS-on-SOI process that has previously been used for fabrication of thick disk resonators [4,5] was used for fabrication of the resonators in this work. Figure 1 shows the SEM view of a fabricated $20 \mu \mathrm{m}$ thick, $40 \mu \mathrm{m}$ wide, $150 \mu \mathrm{m}$ long clamped-clamped SiBAR with $170 \mathrm{~nm}$ capacitive gaps. The resonators are expected to operate in their first width extensional modes, and the operating frequencies are determined by their width.

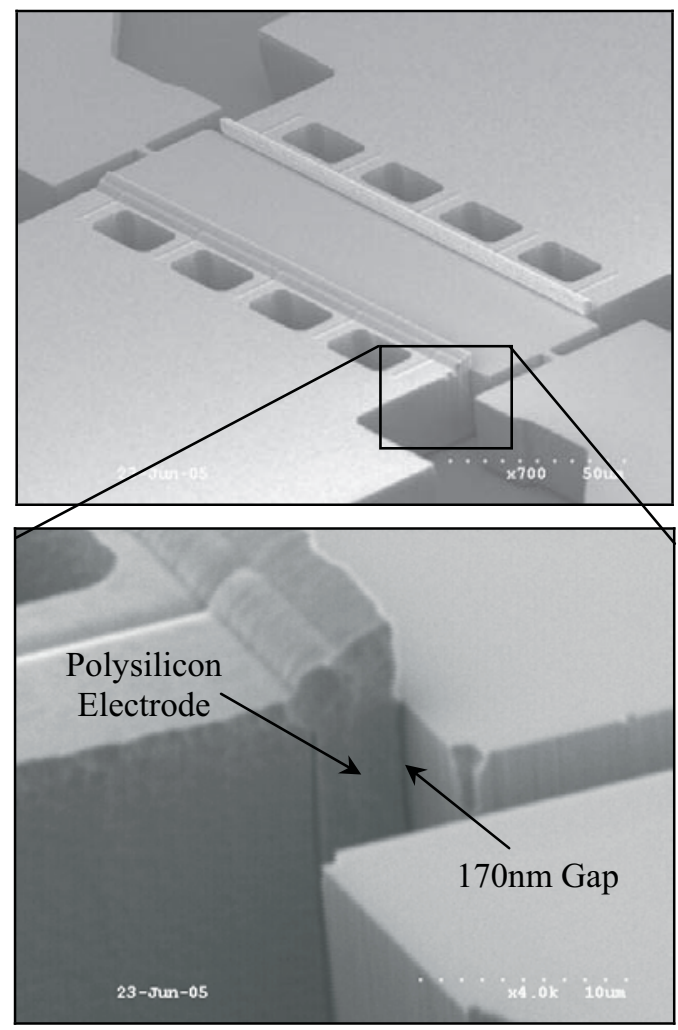

Figure 1. SEM view of a $40 \mu \mathrm{m}$ wide, $150 \mu \mathrm{m}$ long, $20 \mu \mathrm{m}$ thick clamped-clamped SiBAR and the close up of its electrode showing the $170 \mathrm{~nm}$ capacitive gap.

In the previous demonstration of SiBARs, the support lengths were set to the quarter wavelength at the operating frequency (half of the resonator width). This was assuming that such support dimension would minimize flow of energy from the supports to the substrate and maximize the quality factor of the resonator. However, it is shown here that the support length does not have a significant effect on the Q of the clamped-clamped SiBARs and very high quality factors in the same range can be obtained for devices with much shorter supports. The support length for the resonator in Figure 1 is only $4 \mu \mathrm{m}$ making the structure much stiffer and capable of tolerating much larger polarization voltages without getting pulled-in. As a result, over one order of magnitude lower equivalent electrical impedances are achieved for such resonators. 
Figure 2 shows the measured frequency response for the resonator of Figure 1 operating in its first width extensional mode at $107 \mathrm{MHz}$. As shown in Figure 2a, the quality factor of the resonator is 49,600 when biased at a low $V_{p}$. However, it gradually drops to 13,200 as the $\mathrm{V}_{\mathrm{p}}$ increases to $90 \mathrm{~V}$ (Figure $2 \mathrm{~b}$ ). This is due to the existence of an unexpected loading resistance in the resonator structure. The measured impedance with $\mathrm{V}_{\mathrm{p}}$ of $90 \mathrm{~V}$ is $1050 \Omega$. The real motional resistance of the resonator can be back calculated using the unloaded $Q$ value measured at low $V_{p}$ (before the $\mathrm{Q}$ loading starts) and the following equations:

$$
R_{\text {meas }}=R_{m}+R_{\text {load }}, \text { and } \frac{Q_{\text {loaded }}}{Q_{\text {unloaded }}}=\frac{R_{m}}{R_{\text {meas }}}
$$

Where $R_{m}$ is the motional resistance of the resonator, $R_{\text {meas }}$ is the resistance measured by the network analyzer, and $R_{\text {load }}$ is the loading resistor. Motional resistance of $\underline{\mathbf{2 8 0} \Omega}$ is extracted from the resonator responses in Figure 2.

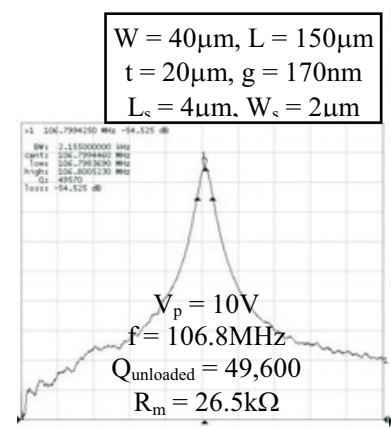

(a)

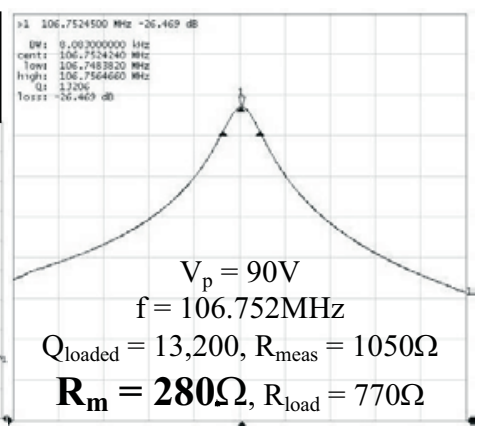

(b)
Figure 2. Measured frequency response of the SiBAR of Figure 1 showing Q loading due to extremely low resonator motional resistance.

Figure 3 shows a similar set of measured frequency responses for a $50 \mu \mathrm{m}$ wide, $300 \mu \mathrm{m}$ long SiBAR fabricated on the same substrate. The extracted motional resistance for this resonator is $\underline{200 \Omega}$.

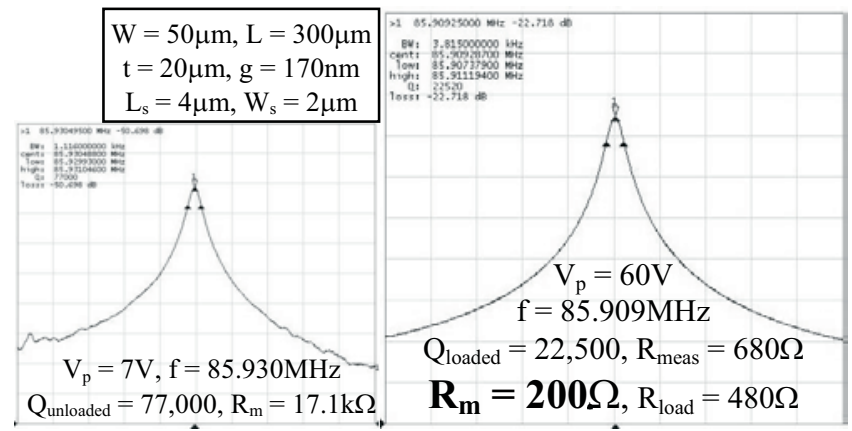

Figure 3. Measured frequency response of a $50 \mu \mathrm{m}$ wide, $300 \mu \mathrm{m}$ long SiBAR with motional resistance as low as $200 \Omega$.

The measured static resistances between the two polarization voltage pads on the two sides of the resonators are close to the extracted loading resistor values. Therefore, the resistivity of the silicon substrate in the $V_{p}$ path is suspected to be responsible for $Q$ loading.

The starting SOI substrate for the resonators in Figures 2 and 3 had a device layer resistivity of $0.015 \Omega$.cm. To further investigate the effect of substrate resistivity on Q loading, another batch of resonators were fabricated on a SOI substrate with lower device layer resistivity $(0.002 \Omega . \mathrm{cm})$. Figure 4 shows the frequency response and extracted resistance values for a $20 \mu \mathrm{m}$ thick, $40 \mu \mathrm{m}$ wide, $150 \mu \mathrm{m}$ long SiBAR with $125 \mathrm{~nm}$ capacitive gaps (Gap aspect ratio $=160)$ fabricated on the lower resistivity substrate. The extracted loading resistance for this resonator is only $120 \Omega$ which is over 6 times lower than the loading resistor for the same device on the previous higher resistivity substrate.

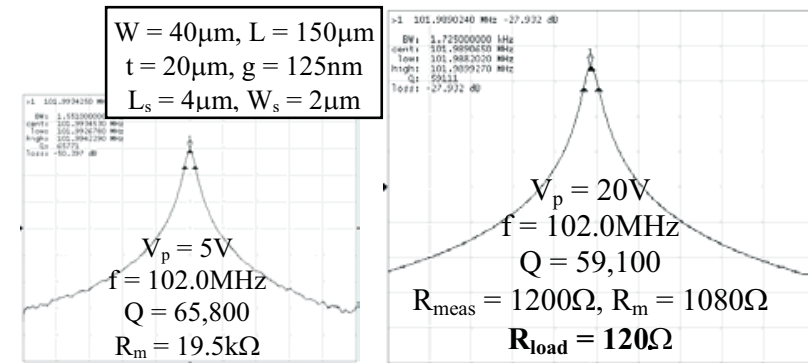

Figure 4. Frequency response of a $\mathrm{SiBAR}$ with similar dimensions as the resonator of Figure 2, fabricated on a lower resistivity substrate showing a much lower loading resistance of $120 \Omega$.

\section{Frequency Tuning and Temperature Compensation:}

One of the remaining major bottlenecks limiting the capability of silicon resonators is their much larger temperature coefficient of frequency compared to quartz resonators. This results from larger temperature coefficient of young's modulus of silicon. Figure 5 shows the measured frequency response as well as temperature dependant frequency drift graph for a $50 \mu \mathrm{m}$ wide, $540 \mu \mathrm{m}$ long SiBAR. The measured frequency drift of $-27.8 \mathrm{ppm} /{ }^{\circ} \mathrm{C}$ is in good compliance with previously reported values for single crystal silicon resonators [4]. At lower frequencies electrostatic frequency tuning of the capacitive resonators can be large enough for temperature compensation over $\mathrm{a}>150^{\circ} \mathrm{C}$ range [1]. However for frequencies in the $100 \mathrm{MHz}$ and above, the electrostatic tuning is not large enough for temperature compensation unless extremely narrow (sub-100nm) capacitive gaps are realized and/or comparatively large polarization voltages are applied.
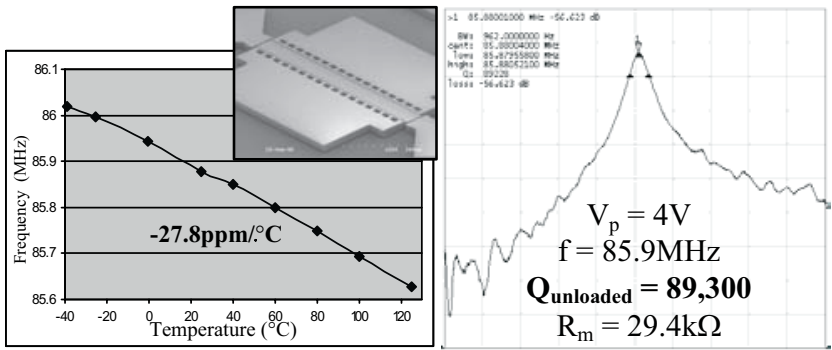

Figure 5. SEM view, frequency response and temperature induced frequency drift data for a $20 \mu \mathrm{m}$ thick, $50 \mu \mathrm{m}$ wide, $540 \mu \mathrm{m}$ long SiBAR with $170 \mathrm{~nm}$ gaps.

The alternative frequency tuning approach evaluated in this work is using the large temperature drift of the resonator for its tuning as well as temperature compensation at the cost of burning extra power. Conductivity of the body of the resonators and availability of two pads on the two end of the device make it very convenient to use the resonator itself as a heater. Figure 6 shows a frequency tuning of more than $1 \mathrm{MHz}(1.2 \%)$ achieved for the $86 \mathrm{MHz}$ resonator of Figure 5 by passing a DC current through its body. This is done by applying slightly different bias voltages to the two $\mathrm{V}_{\mathrm{p}}$ pads of the resonator. According to the measured temperature induced frequency drift (Fig. 5), this is equivalent to 
an elevated temperature of $\sim 450^{\circ} \mathrm{C}$ for the resonator (neglecting second order effects). It is worth noting that the resonator was operating flawlessly with no degradation of the quality factor at high temperature. A temperature dependant current source can be used to keep the temperature of the resonator elevated (e.g. at $125^{\circ} \mathrm{C}$ ) independent of the temperature of the surrounding environment and avoid the temperature drift. In this case, the unpackaged resonator on a $1.2 \times 1.2 \mathrm{~cm}^{2}$ silicon substrate with no thermal isolation from the test setup, $85 \mathrm{~mW}$ of power was required at room temperature to keep the temperature of the resonator elevated at $125^{\circ} \mathrm{C}$. This can be reduced to a few $\mathrm{mW}$ or even below $\mathrm{mW}$ for a single resonator inside a thermally isolated package.

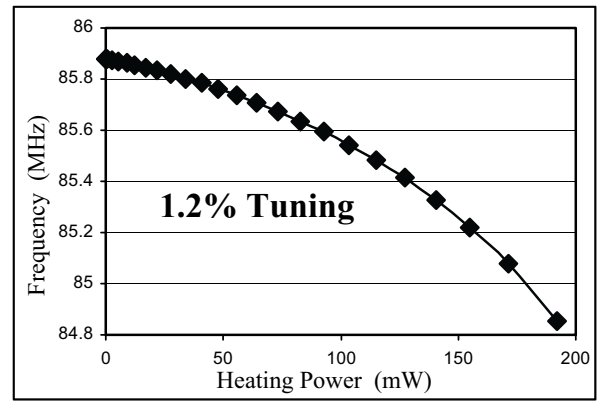

Figure 6. Measured frequency tuning characteristic of the resonator of Figure 5 resulting from heating current passed through the resonator.

Furthermore, one can take advantage of temperature dependency of the resistance of the resonator and use it as a simultaneous in-situ temperature sensor. Incorporation of an active feedback mechanism around such device can result in a highly stable temperature characteristics.

\section{Higher resonance modes:}

Figure 7 shows the measured resonance frequency of a $5 \mu \mathrm{m}$ thick, $30 \mu \mathrm{m}$ wide, $150 \mu \mathrm{m}$ long, SiBAR with $135 \mathrm{~nm}$ capacitive gaps operating in its fundamental and higher modes up to the $5^{\text {th }}$ mode.

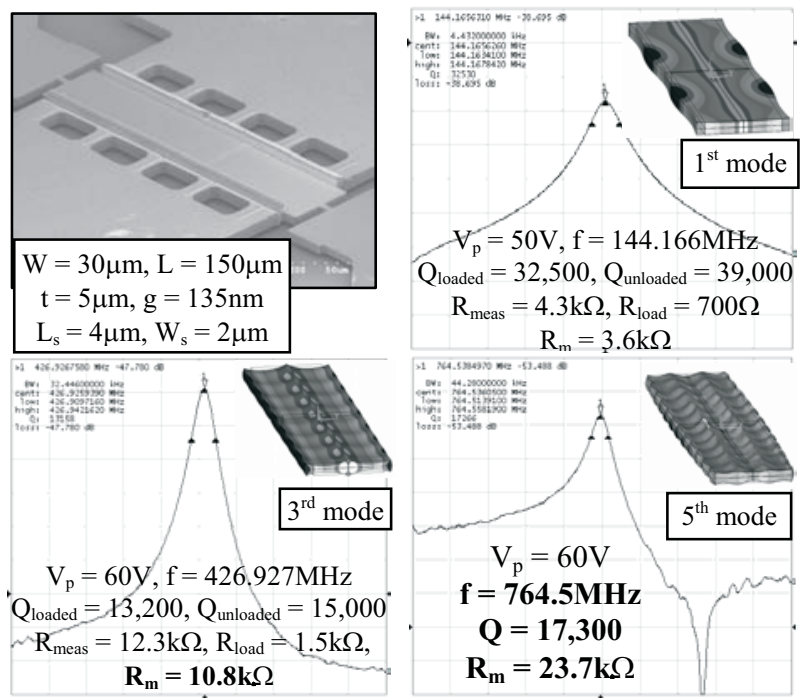

Fig. 7. SEM view and measured frequency response of a $30 \mu \mathrm{m}$ wide, $150 \mu \mathrm{m}$ long, $5 \mu \mathrm{m}$ thick SiBAR at its first and higher odd width extensional modes up to the fifth mode.
The supports for the resonators are in the middle of their width, which is a resonance node for odd higher width extensional modes. Therefore, they can be operated in such higher modes with high quality factors and much higher resonance frequencies.

Quality factors of 15,000 and 17,300 are measured for the $3^{\text {rd }}$ and $5^{\text {th }}$ width extensional modes of this resonator at $427 \mathrm{MHz}$ and $765 \mathrm{MHz}$ respectively. Measured motional resistance of $11 \mathrm{k} \Omega$ and $23 \mathrm{k} \Omega$ for these modes are close to one order of magnitude lower than the impedances reported for surface micromachined capacitive resonators at similar frequencies [6].

\section{ULTRA-LONG SiBARs}

For oscillator applications resonator $\mathrm{Q}$ as high as possible is desired to minimize the close to carrier phase noise. However in most of the filter applications, a comparatively small $(<1000)$ filter $\mathrm{Q}$ is targeted and very high resonator Q can help slightly reduce filter insertion loss, however is not a necessity; instead very low resonator motional resistances are required to avoid the need for large terminating resistors and resulting problems caused by parasitic input and output capacitance of the resonators [7]. In other words, low resonator impedance has to be resulting from strong electromechanical coupling not its high quality factor. In addition to increasing $\mathrm{V}_{\mathrm{p}}$ and reducing the gap size, electromechanical coupling can also be maximized by increasing the length of the resonator. However, elongation of the regular clamped-clamped SiBAR will eventually result in excessive compliance of its structure and consequently stiction and early pull-in issues. To maintain the resonator stiffness while increasing its length, perpendicular support beams are added at several locations along the length of the ultra-long SiBARs (Figure 8).

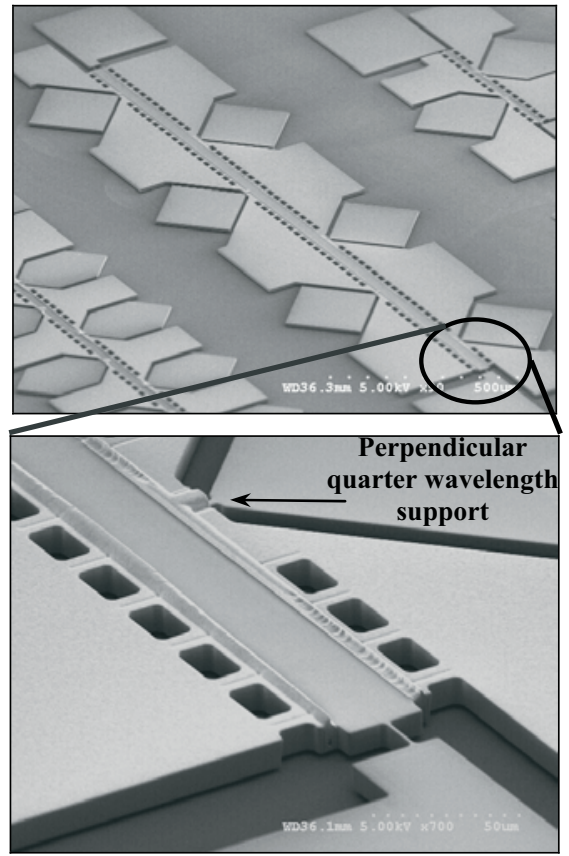

Fig. 8. SEM view of an ultra-long $(30 \mu \mathrm{m}$ wide, $1800 \mu \mathrm{m}$ long) SiBAR with perpendicular supports along its length.

As expected, the added supports reduce the resonator quality factor significantly (Figure 9a). Adding notches to the resonating body at the support interconnects helps increase its quality factor to some extent [6] (Figure 9b). However the Q is still much lower than the regular clamped-clamped SiBAR. Such large structures are very susceptible to having unwanted spurious modes close to their main 
mode (Figure 9). Therefore, precautions need to be taken in their design to suppress the undesired modes as much as possible.

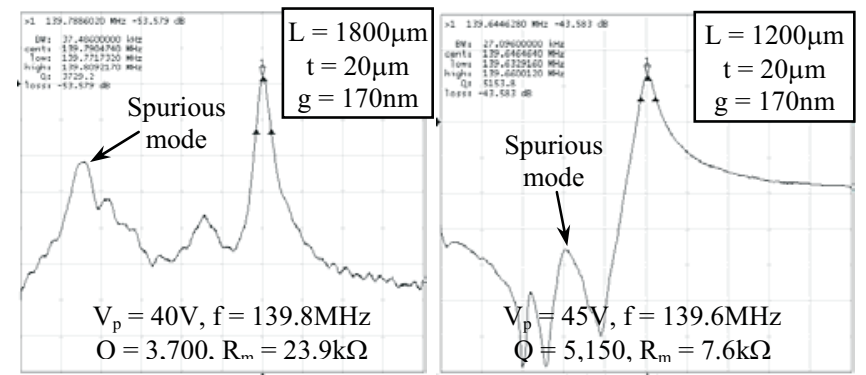

(a)

(b)

Figure 9. Measured frequency response of ultra-long $20 \mu \mathrm{m}$ thick $30 \mu \mathrm{m}$ wide SiBARs with perpendicular support beams along their length, (a) solid (un-notched) BAR, (b) notched BAR.

\section{SQUARE SILICON BARS}

Corner supported square structures are an alternative approach explored in this work to increase the effective resonator length. Figure 10 shows the SEM view and frequency response of a single square SiBAR supported at its four corners. Polysilicon interconnects bridging over the resonator [4] provide electrical connection between different sections of the electrodes for the square SiBARs.

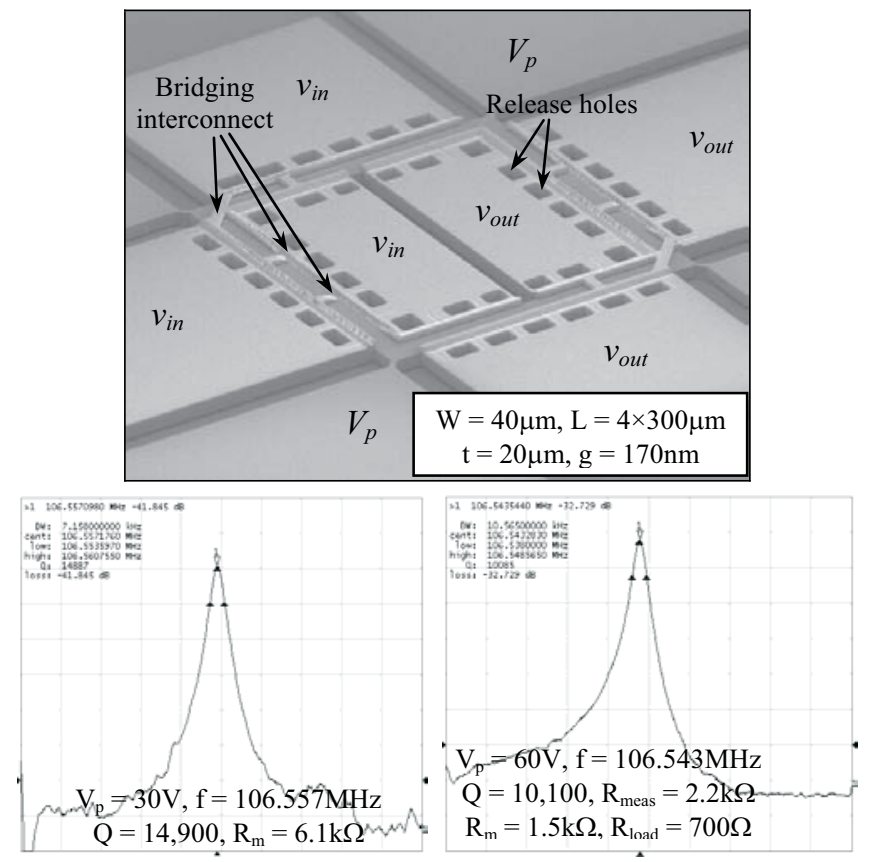

Figure 10. SEM view of a $20 \mu \mathrm{m}$ thick $40 \mu \mathrm{m}$ wide cornersupported square silicon BAR and its measured frequency response under low and high polarization voltages.

In the square resonator of Figure 11 the length maximization is taken another step further. This resonator consists of a network of four squares sharing common sides with each other. Square SiBARs have larger quality factors compared to the linear ultralong SiBARs. However their quality factor is much lower than that of the regular clamped-clamped SiBARs. Extensive finite element analysis and measurements are required for complete characterization and insightful design of the ultra-long linear BARs and the square BARs.

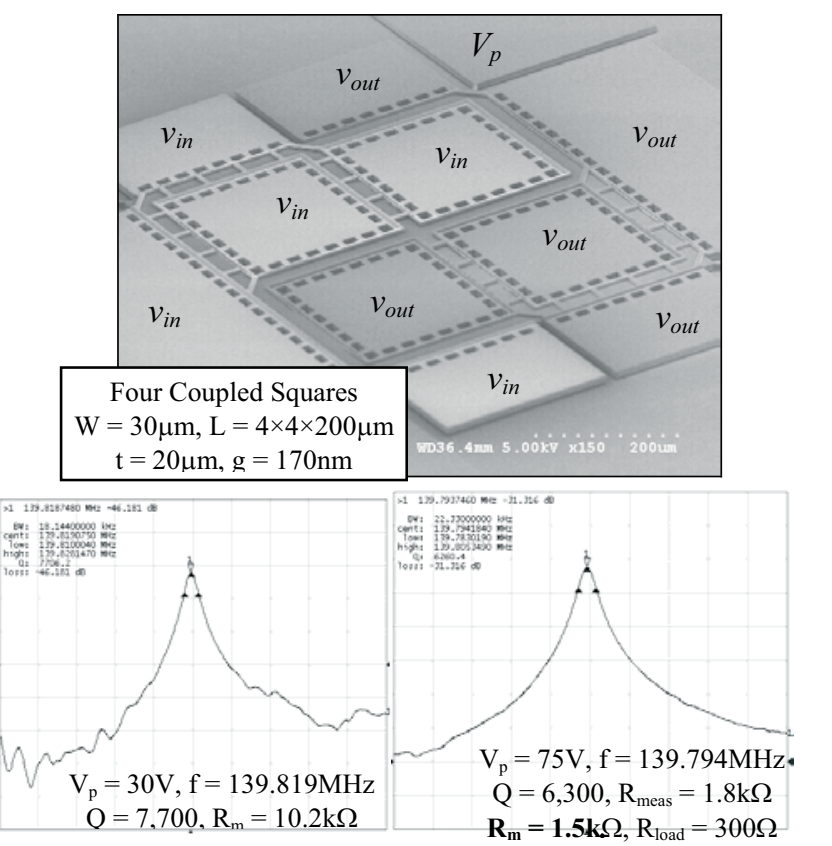

Figure 11. SEM view of a 4-square network silicon BAR and its measured frequency response with different polarization voltages.

\section{CONCLUSIONS}

In conclusion, low impedance high frequency capacitive resonators with great potential for implementation of highly stable low phase noise oscillators are presented. Quality factors as high as a few tens of thousand and motional resistance well below $1 \mathrm{k} \Omega$ are simply achievable for SiBARs in the VHF range without the need for ultra-thin (sub-100nm capacitive gaps).

Poor temperature stability of silicon resonators compared to quartz crystals is the major drawback for employment of such resonators in wide range applications. By passing current through the conductive body of the clamped-clamped SiBARs they can be used as self-heaters as well as temperature sensors simultaneously. An active feedback mechanism along with optimal packaging for thermal isolation enables realization of low power highly temperature-compensated silicon frequency reference.

A variety of ultra-long SiBAR structure can be deployed for improved electromechanical coupling at the cost of reducing the resonator quality factors.

\section{Acknowledgements}

This work is supported under the DARPA NMASP program.

\section{References:}

[1] G.K. Ho, et al, "Temperature compensated IBAR reference oscillators," Proceedings MEMS'06, pp. 910-913.

[2] Y.-W. Lin, et al, " $60-\mathrm{MHz}$ wine glass micromechanical disk reference oscillator," Digest of Technical Papers, ISSCC 2004, pp. 322-323

[3] S. Pourkamali, G. K. Ho and F. Ayazi, "Vertical capacitive SiBARs," MEMS'05, pp. 211-214.

[4] S. Pourkamali, et al, "VHF single crystal silicon side supported disk resonators-Part II: implementation and characterization," JMEMS, Vol. 13, Issue 6, December 2004, pp. 1054-1062.

[5] S. Pourkamali and F. Ayazi, "High frequency capacitive micromechanical resonators with reduced motional resistance using the HARPSS technology," proceedings, $5^{\text {th }}$ Silicon RF topical meeting 2004, pp. 147-150.

[6] S.-S. Li, Y.-W. Lin, Y. Xie, Z. Ren, and Clark T.-C. Nguyen, "Micromechanical hollow-disk ring resonators," MEMS'04, pp. 821-824.

[7] S. Pourkamali and F. Ayazi, "Electrically coupled MEMS bandpass filters-Part I: with coupling element," Journal of Sensors and Actuators A 122, 2005, pp. 307-316. 